\title{
Is it Rheumatic Aortitis, Fatty Streaking or Both?
}

\author{
Shirley Siew
}

Division of Human Pathology, Michigan State University, East Lansing, MI, USA

Rheumatic fever is a systemic disease with prominent involvement of the joints, the heart, the brain and the lungs. While the lesions in the other organs, regress, there is progression of the pancarditis. Valvular deformities are common and rheumatic arterits is a well-recognized entity. Acute parietal endocarditis has been reported [1]. However, little is known about rheumatic aortitis. We report two cases with lesions in the thoracic aorta.

CASE I:- A 14 year old boy presented with a history of breathlessness on exertion since the age of 8 . He did not respond to treatment with digitalis. Three days before admission, he developed a cold with fever, difficulty breathing and pain on the right side of the chest. Pertinent findings at autopsy were gross cardiac enlargement-540gm/Normal 300gm, involving both sdes. The mitral, tricuspid and pulmonary valves were within normal limits. The aortic valve was fibrosed with fusion of the cusps resulting in marked aortic stenosis. The ascending aorta was markedly dilated. There were two raised, irregular colorless plaques above the aortic valve [Fig. 1]. There was both acute and chronic passive congestion of the lungs, slight edema and patchy consolidation. Histopathology of the plaques in the ascending aorta showed the presence of well-marked thickening of the intima and a heavy, diffuse infiltration of nongranular cells [Fig. 2]. There was a paucity of foam cells.

CASE II:- A 23 year old woman presented with a history of a heart murmur since the age of 13. Following acute tonsillitis at the age of 6 , she manifested classical features of rheumatic fever with migratory arthritis and subcutaneous nodules. Pertinent cardiac findings on admission were the presence of mitral stenosis and aortic regurgitation. An open commissurotomy (splitting of the commissures) was performed and the aortic valve was replaced by a bioprosthesis. The patient was readmitted to hospital, 5 weeks after surgery with a history of fever and chills for the preceding 3-6 days. The hospital course was stormy with continued periods of spiking temperatures, going up to $105 \mathrm{~F}$, despite the administration of massive antibiotics. On the $8^{\text {th }}$ hospital day, the patient had the sudden onset of marked sweating, shortness of breath, chest pain and an ashen grey complexion. She sustained a cardiopulmonary arrest and could not be resuscitated. During this hospital stay, eight out of eight blood cultures that were performed showed the presence of Staphylococcus epidermidis. Pertinent findings at autopsy were the presence of acute bioprosthetic endocarditis with large vegetations (septic thrombi) on the prosthetic cusps, impacting upon the aortic valve orifice. An embolus occluded the proximal portion of the left circumflex artery. There was a commissurotomy of the mitral valve, the cusps were thickened and platelet vegetations were present. There was thickening, fusion and shortening of the chordae tendineae. There was recent infarction of the spleen and the kidneys. Gross examination of the aorta showed the presence of fatty streaking, most marked in the descending aorta [Fig. 3].

Histopathology confirmed the presence of infective endocarditis and acute rheumatic pancarditis. There was thickening of the intima of the aorta with a dense infiltration of non granular cells and elongated histiocytes. Variable numbers of foam cells were seen at the different levels [Fig.4].

The plaques above the aortic valve in Case I are quite distinct from atherosclerosis and there are few 
foam cells in the histopathologic findings, which are in keeping with those of acute rheumatic aortitis. Grossly, there is fatty streaking in the aorta in Case II. But, histologically, the reaction is mainly that of non granular cells and elongated histiocytes with varying numbers of foam cells. It is considered that the complication of acute infective bioprosthetic endocarditis superimposed on acute rheumatic pancarditis precipitated the onset of atherosclerosis with the development of fatty streaking in addition to acute rheumatic aortitis.

References:

[1] S Siew, Rheumatic Heart Disease Revisited:Acute Parietal Endocarditis-Another Piece of the Unsolved Puzzle_Microsc.Microanal. 1522-1523,2008.

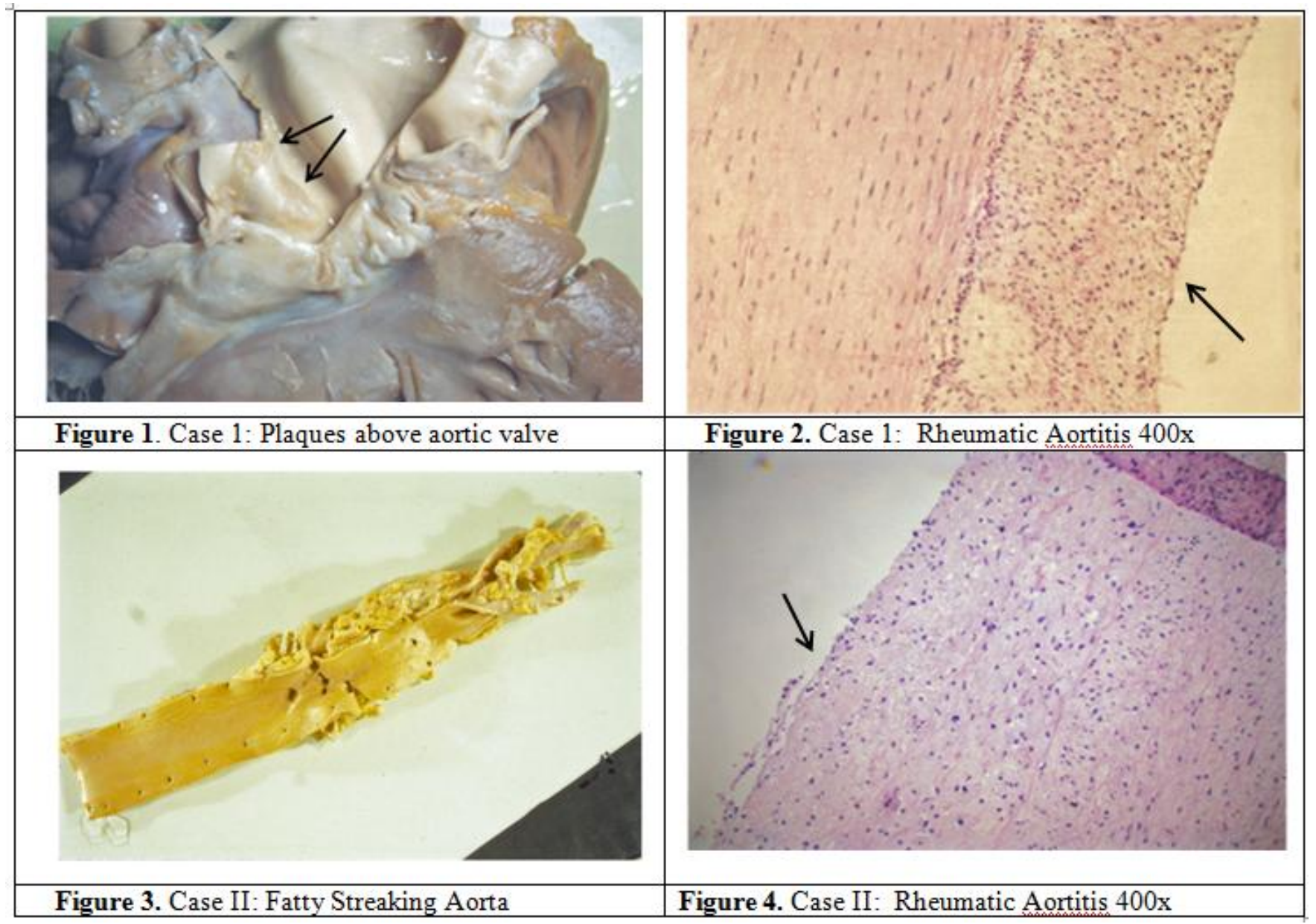

